\title{
Simulation of the plasma-wall interaction in a tokamak with the Monte Carlo code ERO-TEXTOR
}

\author{
A. Kirschner ${ }^{\mathrm{a}}$, V. Philipps ${ }^{\mathrm{a}}$, J. Winter ${ }^{\mathrm{b}}$, U. Kögler ${ }^{\mathrm{a}}$ \\ a Institut für Plasmaphysik, Forschungszentrum Jülich GmbH, \\ Euratom Association, Trilateral Euregio Cluster, \\ Jülich, Germany \\ b Institut für Experimentalphysik II, Anwendungsorientierte Plasmaphysik, \\ Ruhr-Universität Bochum, \\ Bochum, Germany
}

\begin{abstract}
The interaction of plasma with the walls has been one of the critical issues in the development of fusion energy research. On the one hand, plasma induced erosion can seriously limit the lifetime of the wall components, while, on the other hand, eroded particles can be transported into the core plasma where they lead to dilution of the fusion plasma and to energy losses due to radiation. Low- $Z$ wall materials induce only small radiation losses in the plasma core but suffer from large physical sputtering rates. Carbon based materials in addition suffer from chemically induced erosion. High- $Z$ wall materials show significantly smaller erosion but lead to large radiation losses. One of the main goals of present plasma-wall studies is to find a special choice of wall materials for steady state plasma scenarios that will provide an optimum with respect to fuel dilution, radiation losses, wall lifetime and fuel inventory in the walls. To obtain a better understanding of the processes and to estimate the plasma--wall interaction behaviour in future fusion devices the 3-D Monte Carlo code ERO-TEXTOR, based originally on the ERO code, has been developed. It models the plasmawall interaction and transport processes in the vicinity of a surface positioned in the boundary layer of TEXTOR. The main aim is to simulate the erosion and redeposition behaviour of different wall materials under various plasma conditions and to compare this with experimental results. This contribution describes the main features of the ERO-TEXTOR code and gives some examples of simulation calculations to illustrate the application of the code.
\end{abstract}

\section{General description}

To describe the erosion and deposition processes in the vicinity of a limiter in the boundary layer of magnetically confined fusion plasmas various processes have to be taken into consideration. The EROTEXTOR code, based originally on the ERO code [1], treats these processes as follows.

First, the impinging background plasma ions (fuel and impurities) can erode particles from the limiter surface by physical and chemical sputtering. The released particles leave the limiter as neutrals. It is assumed that the neutrals do not interact with the plasma particles so that they move along straight lines. While moving as neutrals through the plasma at each time step the ionization probability is calculated, which depends on the local plasma parameters (electron density and temperature) and a probability function taking into account the statistical nature of ionization. Thus, the particles are ionized at some distance from their erosion site. The ions are acted on by the magnetic and electric fields. In addition, the charged particles interact with the background plasma through collisions and can be further ionized to higher charge states. The originally eroded particles now have a certain probability to return to the limiter surface - of course in general this location is not identical with the point of production. For the returning particles the reflection coefficient is calculated using the TRIM database and the probability of redeposition is obtained according to this value. If a particle is not redeposited it will again move into the plasma as a neutral and the above procedure starts again until the particle is redeposited or leaves the observation volume. The dimensions of the observation volume around the limiter are determined by the user of the code. If it is chosen large enough, the probability that a particle leaving this volume returns to the surface is small and therefore the error made in neglecting these particles is also small.

The code can take into account the deposition of carbon ions from the background plasma onto the limiter surface assuming a certain impurity flux ratio 


\section{A. Kirschner et al.}

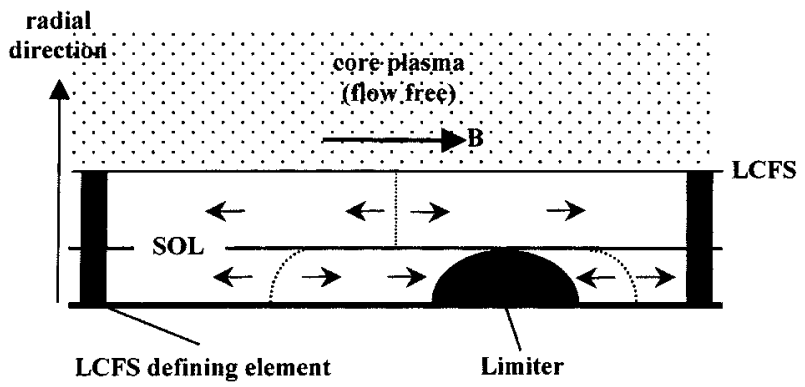

Figure 1. Modelling geometry for the ERO-TEXTOR code. The diagram shows the case where the test limiter (here, as an example, spherically shaped) is not the LCFS defining wall element so that the SOL divides into three parts. The dotted lines represent the local stagnation points, i.e. locations where the plasma velocity is zero. The arrows inside the SOL indicate the local direction of the plasma velocity $c_{s}(s)$. The co-ordinate $s$ is the distance to the stagnation point along the magnetic field $B$.

which has to be determined by the user as input parameter from the experimental data. After having determined the reflection coefficient of the arriving carbon ions the amount of deposition can be calculated.

\section{Description of the background plasma}

\subsection{Plasma flow}

The background plasma used in the EROTEXTOR code is divided into the SOL plasma which exists outside the LCFS of the magnetic field and the confined plasma inside the LCFS. The LCFS is defined either by the test limiter itself, if it is positioned at smaller minor radius than other TEXTOR wall elements or by other limiters if the test limiter is positioned at larger radii. The connection lengths to the different wall elements from the electron and ion drift sides have to be given as input parameters. Figure 1 shows as an example the situation, where the test limiter is not the LCFS defining element.

The confined plasma is assumed to have no net flow (plasma flow velocity zero). In contrast, a plasma flow establishes inside the SOL. It is produced by absorption of the plasma by the limiting wall elements. This produces a flow parallel to the magnetic field, $\Gamma_{\|}(r, s)$ ( $r$ is the radial co-ordinate and $s$ the co-ordinate parallel to the magnetic field with $s=0$ at the stagnation point between the limiter and the limiting wall elements where the plasma velocity is zero). A one dimensional plasma model $[2,3]$ is used to calculate the velocity $c_{S}(r, s)$ of this flow. This model also calculates the dependence of the electron density $n_{e}(r, s)$ along the magnetic field. The radial dependence of the plasma flow density $\Gamma_{\|}(r, s)=n_{e}(r, s) c_{S}(r, s)$ is obtained by assuming an exponential profile of the electron density in the radial direction, as described in the following section.

\subsection{Plasma temperature and density}

The radial dependences of the electron density $n_{e}$ and electron and ion temperatures $T_{e}, T_{i}$ are assumed to be exponential,

$y(r)=y\left(r=r_{L C F S}\right) \exp \left[-\left(r_{L C F S}-r\right) / \lambda_{y}\right]$

with $y=\left\{T_{e}, T_{i}, n_{e}\right\}$. The decay lengths $\lambda_{y}$ as well as the density and temperatures at the LCFS are determined by input parameters which are taken from experimental data. As mentioned already the absorption of the plasma by the wall produces a decreasing electron density along the magnetic field lines towards the wall element. The density dependence parallel to the field lines is given by $[2,3]$

$n_{e}(s)=n_{e}(s=0) \frac{s^{2}}{L_{C}} \frac{1}{L_{C} / 2-\sqrt{\left(L_{C} / 2\right)^{2}-s^{2}}}$

where the connection length $L_{C}$ is an input parameter of the ERO-TEXTOR code and can have different values inside different regions of the SOL (Fig. 1). According to Eq. (2) $n_{e}(s)$ becomes $n_{e}(s=0)$ for $s \rightarrow 0$ and $0.5 n_{e}(s=0)$ for $s \rightarrow L_{C} / 2$, where $s=L_{C} / 2$ corresponds to the location of the limiter surface. The input parameter $n_{e}\left(r=r_{L C F S}\right)$ in (1) is the value of the electron density at $r=r_{L C F S}$ and $s=0$.

From the density and temperature profiles the particle flux density $\Gamma_{P}$ of the species $P$ (impurity or fuel ions) arriving at the limiter surface is calculated according to

$\Gamma_{P}=n_{e}(r, s) c_{S 0}(r) f_{P} \cos \alpha$

with $c_{S 0}(r)=\left\{k\left[T_{e}(r)+T_{i}(r)\right] / m_{i}\right\}^{1 / 2}$, where $c_{S 0}$ is the ion acoustic speed of the fuel ions of mass $m_{i}$, $f_{P}$ the relative concentration of the species $P$ in the plasma and $\alpha$ the angle between the magnetic field lines and the surface normal. Because of cross-field diffusion, gyromotion and - most important - local radial electric fields, the particle flux $\Gamma_{P}$ is not equal to zero for magnetic field lines parallel to the limiter surface or at shallow impact angles. Therefore a cut-off angle of $80^{\circ}$ (somewhat arbitrary) is used in 
the ERO-TEXTOR code such that for larger impact angles the impinging particle flux is kept constant at the value reached for an impact angle of $80^{\circ}$.

Hydrogen, deuterium and tritium can be used as plasma fuel ions. Several impurities, such as helium, carbon, oxygen and neon, are implemented into the ERO-TEXTOR code. The user determines the percentage amount of the different impurities at the LCFS, its exponential decay length in the radial direction and its charge state.

\subsection{Electric fields}

Inside the SOL the electric field $\boldsymbol{E}$ consists of three different contributions: the sheath field $\boldsymbol{E}_{\text {sheath }}$, a parallel electric field along the field lines $\boldsymbol{E}_{\text {para }}$ and a radial electric field $\boldsymbol{E}_{\text {rad }}$.

The sheath field is caused by the sheath potential $\phi_{\text {sheath }}, \boldsymbol{E}_{\text {sheath }}=-\boldsymbol{\nabla}\left(\Phi_{\text {sheath }}\right)$. It is perpendicular to the limiter surface and directed towards it so that positive ions are accelerated towards the limiter. The magnitude of the sheath potential is given by [4]:

$$
\begin{aligned}
\Phi_{\text {sheath }}= & -\left(k T_{e} / 2 e\right) \ln \left\{2 \pi\left(m_{e} / m_{i}\right)\left(1+T_{e} / T_{i}\right)\right. \\
& \left.\times\left[1 /\left(1-\gamma^{2}\right)\right]\right\}
\end{aligned}
$$

where $m_{i}$ is the mass of the plasma species, $m_{e}$ the electron mass, $T_{e}$ the electron temperature, $T_{i}$ the ion temperature and $\gamma$ the secondary electron emission coefficient of the limiter material. The radial extension of the sheath depends on the direction of the magnetic field relative to the limiter surface. In dependence of the angle $\alpha$ between the magnetic field and the surface normal, the sheath is divided into an electric sheath $\Phi_{S}$ and a magnetic presheath $\Phi_{m p s}$ $[5,6]$,

$\Phi_{S}=\Phi_{\text {sheath }} f_{d}(\alpha)$ and $\Phi_{m p s}=\Phi_{\text {sheath }}\left[1-f_{d}(\alpha)\right]$

where $f_{d}(\alpha)$ is the so-called Brooks parameter [6] which approaches a value of one for $\alpha \rightarrow 0^{\circ}$ (in this case the sheath is dominated by the electric part) and a value of zero for $\alpha \rightarrow 90^{\circ}$ (domination of the magnetic part). The radial extension of the sheath is then given by

$\Phi(u)=\Phi_{S} \exp \left(-u / 2 \lambda_{D}\right)+\Phi_{m p s} \exp \left(-u / R_{L}\right)$

where $u$ is the normal distance from the limiter surface, $\lambda_{D}$ the Debye length and $R_{L}$ the gyration radius of the plasma ions. Because $R_{L}$ is typically in the range of a few millimetres or even smaller and $\lambda_{D}$ is not larger than $\sim 10 \mu \mathrm{m}$, the sheath field exists only in a thin layer above the limiter surface.
The parallel electric field $\boldsymbol{E}_{\text {para }}$ originates from the gradient of the electron density along the magnetic field inside the SOL (Section 2.1) and is thus parallel to the magnetic field lines. As described in Ref. [7] one obtains

$E_{\text {para }}(r, s) \propto-\frac{T_{e}(r)}{n_{e}(r, s)} d n_{e \|}(s) / d s$

Moving from the stagnation point towards the limiter, the electron density decreases but its gradient, $d n_{e \|}(s) / d s$ increases. These two counteracting effects result in a parallel electric field $E_{\text {para }}$, which increases with increasing $s$. The gradient $d n_{e \|}(s) / d s$ points away from the limiter so that, according to (6), $E_{\text {para }}$ is directed towards the limiter. Within the sheath the parallel field is much smaller compared with $E_{\text {sheath }}$.

Finally, the code includes a radial electric field $E_{\text {rad }}$ in the SOL outside the LCFS. This is caused by the electric potential of $E_{\text {para }}$. Since the electron temperature decreases radially the potential of $E_{\text {para }}$ decreases and a radial electric field is generated,

$E_{r a d}(r, s) \propto f(s) d T_{e}(r) / d r$, with $f(s) \leq 0$.

$d T_{e}(r) / d r$ points in the direction of the plasma centre from which it follows that $E_{r a d}(r, s)$ is directed to the wall and thus also towards the limiter surface. Inside the sheath, however, $E_{\text {rad }}$ is small compared with $E_{\text {sheath }}$. The radial field decreases itself with increasing distance from the limiter along the magnetic field. This dependence is described by the function $f(s)$ and has a scale length of the order of the connection length $L_{C}$ ( $\sim$ metres).

Figure 2 compares the magnitude of the different electric fields inside the SOL using typical plasma parameters for TEXTOR. The parallel and radial electric fields vanish inside the LCFS, and thus inside the core plasma. Thus within the present model only the sheath field due to the sheath potential remains inside the LCFS.

\section{Interaction between the background plasma and the limiter surface}

\subsection{General}

The ERO-TEXTOR calculations are divided into time steps whereby the length of the time steps and the overall number of time steps (total simulation time) is chosen by the user. During each time step 


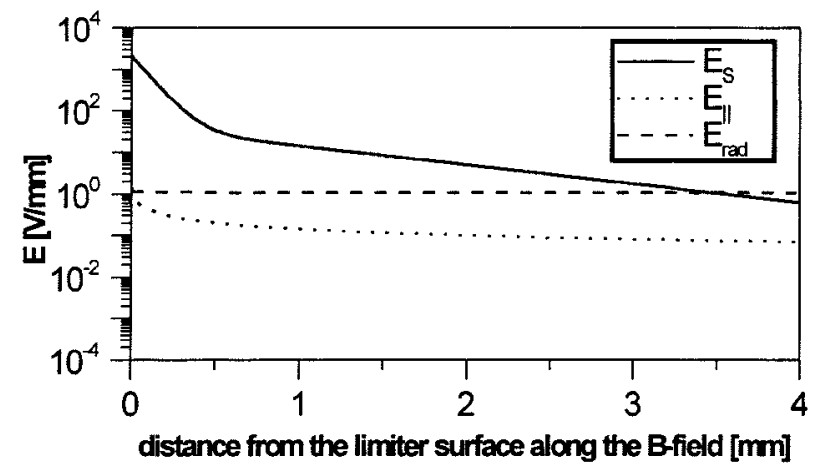

Figure 2. Comparison of the different electric fields inside the plasma as a function of the distance from the limiter surface along the magnetic field. The fields are calculated assuming the following plasma parameters: $T_{e, i}=40 \mathrm{eV}, \lambda_{T_{e, i}}=25 \mathrm{~mm}, L_{C}=11 \mathrm{~m}$ and $60^{\circ}$ for the angle between the magnetic field and the surface normal.

the composition of the limiter surface is kept constant. After the erosion and deposition has been calculated within a given time step the new elemental composition of each surface element is determined. This new surface composition is then used as input for the next simulation step.

The spatial resolution on the limiter surface is determined by a net over the surface whereby the area of the cells $A_{\text {cell }}$ is determined via the input parameters. Here a compromise has to be found between sufficient spatial resolution on the limiter surface and the computational time.

\subsection{Physical erosion}

Impact of plasma ions and impurities can lead to physical sputtering of material from the limiter surface. The sputtering yield of the species $X$ from the surface by the impact of particles $P$ from the background plasma, $Y_{\text {phys }}^{P \rightarrow X}\left(E_{0}, \alpha_{0}\right)$, is calculated according to formulas of Bohdanski and Yamamura [8, 9]. However the impact energy of the plasma ions is not monoenergetic and particles hit the surface at different impact angles. To take such effects into account the ERO-TEXTOR code uses an averaged sputtering yield, $\bar{Y}_{p h y s}^{P \rightarrow X}$, as derived by Abramov et al. [10]. In the Abramov approximation the yield is obtained by averaging the sputtering yields $Y_{p h y s}^{P \rightarrow X}\left(E_{0}, \alpha_{0}\right)$, over the energy and angle of incidence of the incoming particles. The averaging is based on the assumption that the background plasma ions far away from the limiter have a Maxwellian energy distribution which results in a 'shifted Maxwellian' energy distribution after the acceleration in the sheath potential $\Phi_{\text {sheath }}$. It should be noted that the sheath does not change the Maxwellian energy distribution [10]. The resulting averaged sputtering yield $\bar{Y}_{p h y s}^{P \rightarrow X}$ depends then on the local electron and ion temperatures $T_{e}$ and $T_{i}$ and on the charge state of the background ions, which has to be given as an input parameter of the ERO-TEXTOR code. The amount of sputtered particles $X$ from each surface cell, $N_{X}$, is given by

$N_{X}=c_{X} \Gamma_{P} \bar{Y}_{p h y s}^{P \rightarrow X} A_{c e l l} \Delta t$

where $c_{X}$ is the time dependent concentration of the element $X$ in the surface cell, $\Gamma_{P}$ the incoming flux of particles $P$ to the cell (according to (3)) and $\Delta t$ the time step of the simulation.

All sputtered particles leave the limiter surface as neutrals. Their energy distribution is given by the Thompson distribution [11] around the surface binding energy. At present, for simplification and because of lack of knowledge, the binding energies of the pure materials are used (independent of the surface composition). Thus no real 'chemical' formation of a new type of material with different binding energies is taken into account. The angular direction of the sputtered particles is given by a cosine distribution around the surface normal.

\subsection{Chemical erosion}

Chemical erosion of carbon by hydrogen and oxygen impact forming volatile hydrocarbons and carbon oxides is a very significant contribution to the erosion of carbon materials. In the present EROTEXTOR calculations, chemical hydrocarbon formation is included only via the formation of the methane molecule $\mathrm{CH}(\mathrm{D}, \mathrm{T})_{4}$ by hydrogen species (i.e. hydrogen, deuterium or tritium). To treat this erosion in the code, either a constant erosion yield is assumed or, alternatively, the erosion yield $Y_{\text {chem }}\left(T, Y_{\text {phys }}, \Gamma\right)$ is given by a formula according to Roth [12]. This formula calculates methane formation depending on the surface temperature, the impinging hydrogen flux density and energy and the hydrogen isotope type. Possible chemical sputtering of silicon through hydrogen by the creation of a silane molecule $\mathrm{SiH}(\mathrm{D}, \mathrm{T})_{4}$ can be described by a fixed sputtering yield, given as an input parameter.

In analogy to Eq. (8) the amount of chemically sputtered particles is calculated using $Y_{\text {chem }}$. The energy distribution of the chemically sputtered methane and silane molecules is Maxwellian with the 
surface temperature as mean temperature, and the direction of the emission is, in analogy to physically sputtered particles, determined by a cone around the surface normal.

Chemical erosion of carbon by oxygen forming carbon oxides is at present included in the code as an additional chemical sputtering yield with a constant value of $0.5[13]$

\subsection{Material deposition onto the limiter surface from the background plasma}

Deposition onto the limiter surface can be due either to background plasma impurities or to particles eroded from the limiter surface which are locally redeposited.

The deposition of carbon from the background plasma is given by the carbon flux (which is defined by the user as the relative fraction to the hydrogen flux) and the reflection probability. The individual reflection coefficients on different materials $R_{C \rightarrow X}\left(E_{0}, \alpha_{0}\right)$ are determined by the TRIM code [14]. The resulting overall reflection coefficient $R\left(E_{0}, \alpha_{0}\right)$ is given by $\sum R_{C \rightarrow X}\left(E_{0}, \alpha_{0}\right) c_{X}$, where $c_{X}$ is the concentration of the element $X$ in a given surface cell. For simplification, the energy $E_{0}$ of the incoming carbon ions is calculated as a sum of the thermal energy and the acceleration energy in the sheath potential, and thus does not take into account the energy distribution of the plasma particles. The angle of incidence $\alpha_{0}$ is assumed to be $60^{\circ}[15]$. The total amount of deposited background carbon particles within a given time step is then $\left[1-R\left(E_{0}, \alpha_{0}\right)\right] \Gamma_{C, \text { in }} A_{\text {cell }} \Delta t$.

\section{Motion of particles through the plasma}

\subsection{Particles eroded by the background plasma}

Within the ERO-TEXTOR code a fixed number of simulation particles start into the plasma from each surface cell. Due to the limited computational time, this number is much smaller than the real number of sputtered particles so that the simulation particles are only representative of the real sputtered particles. The number of simulation particles is given as an input parameter, and the user has to consider critically whether the total number of launched particles is sufficient for statistical purposes.
The neutrals starting from the limiter surface by reflection, physical and chemical sputtering do not interact with the plasma background unless they are ionized or dissociated. Thus they move along straight lines. To calculate the ionization or dissociation of neutrals, the ERO-TEXTOR code determines at each computational time step $d t$ the actual position, the local electron density and temperature and, accordingly, the local ionization (dissociation) probability. Here it is necessary to distinguish between atoms and molecules. For atoms the ionization rate coefficient $\langle\sigma v\rangle$ is calculated according to the Bell formula [16]. The ionization time $t_{i o n}$ and the ionization probability $P_{i o n}$ at each location are given by

$t_{i o n}=\left(\langle\sigma v\rangle n_{e}\right)^{-1}$ and $P_{i o n}=1-\exp \left(-d t / t_{i o n}\right)$

where $d t$ must be kept small compared with $t_{i o n}$. In order to account for the statistical nature of the ionization process the value of $P_{i o n}$ is compared with a random number $x_{\text {ran }}$ (between 0 and 1) and if the condition $P_{i o n}>x_{\text {ran }}$ is fulfilled the particle is ionized. During the following motion as an ion the procedure is repeated at each computational step and the particle can in this way be ionized to higher charge states.

In principle, the released methane and silane molecules are treated in the same way. However, these molecules can undergo many more different processes than just simple ionization. The rate coefficients for the different molecular processes for methane are calculated according to Ehrhardt and Langer data [17]. For silane the same values are used except for some rate coefficients where literature data could be found [17-19]. In a similar way as for the ionization process, it is decided at each computational time step, again with the aid of a random number, whether a dissociation event takes place at all. If this is the case, the decision as to which of the different possible dissociation processes occurs, is made by comparing the individual dissociation times.

When a particle (atom or molecule) is charged, its movement is determined predominantly by the electric and magnetic forces acting on it. The mathematical calculation of the movement of the charged particles through an electromagnetic field is treated in the code by means of the Boris method [20]. This method considers effects like gyration or $\boldsymbol{E} \times \boldsymbol{B}$ drifts automatically.

In addition to electric and magnetic forces the charged particle interacts also with the background plasma ions - opposite to the neals. The interaction of charged particles with background 


\section{A. Kirschner et al.}

plasma ions in the ERO-TEXTOR code consists in principle of three contributions: friction between the particles and the background ions, thermal forces and cross-field diffusion. The friction between the ions produced at the limiter and the background plasma ions is treated by the Fokker-Planck [21] method. The Fokker-Planck equations are derived by solving the Boltzmann equation (including a Coulomb collision term) for a one particle distribution function, which describes a moving particle. The equations deliver three relaxation times, the socalled Spitzer time constants [22], giving the basis to calculate the friction force. The thermal forces [23] are caused by the change of the mobilities of the released particles along the magnetic field lines due to the gradients in the electron and ion temperatures. These forces act on the ions in the direction of higher plasma temperatures and tends to draw them away from the surfaces. The transport of ions across the magnetic flux surfaces is described by a diffusion constant, which has to be given as an input parameter.

Basically, there exist two possible destinies for eroded particles from the limiter. First, a particle can leave the defined observation volume around the limiter. In this case the particle is lost for the simulation. Second, it can return to the limiter surface. In this case the particle itself can erode particles from the limiter surface by sputtering. Since the code knows the individual energy and angle of incidence of the returning particle, the individual sputtering yield can be calculated according to the Bohdanski and Yamamura formulas (opposite to the sputtering by background plasma particles where an integrated sputtering yield is used, Section 3.1). The particles sputtered in this way are emitted again into the plasma and the procedure described above begins anew.

The destiny of the returned particle itself depends on the local reflection probability: it can be either deposited onto the surface, then the simulation ends for this particle, or be reflected as a neutral into the plasma. The reflected particles are neutralized on the surface and move as neutrals through the plasma as described above but with higher starting energies. For atoms the reflection probability and the energy and angle of emission of the reflected atoms are calculated using TRIM data. This procedure is carried out for atoms returning to the surface but cannot be used for molecules returning to the surface due to the lack of reflection data. Thus the user has to define for each molecular species the reflection probability, the energy distribution of the reflected molecules and the molecular state in which the molecule is reflected (i.e. as a radical which hits the surface or as a saturated molecule). The direction of reflection is calculated assuming an emission into a cone relative to the surface normal.

\subsection{External particle source}

To study the local transport of particles near limiter surfaces, experiments were carried out [24] in which particles were puffed through holes in the test limiter surface. The advantage of such a type of experiments is that a well defined amount of gaseous particles (e.g. silane or methane) is puffed under welldefined geometrical conditions.

The ERO-TEXTOR code can be used to simulate such gas puffing experiments. The parameters of the particle source (which kind of particles, energy distribution, angle of emission) are determined by the input parameters and can thus be adapted to the experimental data. The motion of the externally sourced particles and their interaction with the limiter is handled in the same way as described in Section 4.1.

\section{Output information from ERO-TEXTOR simulations}

\subsection{Surface related information}

For each of the surface cells the amount of erosion and redeposition is calculated for each time step. For redeposition the program distinguishes promptly redeposited particles from the rest of the redeposited particles. Promptly redeposited particles are defined as those which are redeposited during their first gyration. For erosion the program distinguishes between particles sputtered by the background plasma flux of hydrogen and impurities and particles eroded by the redeposited particles. The program also calculates the local amount of carbon deposition from the background plasma. Finally the composition of the interaction layer which is reached after the individual time step is determined.

Since the data are calculated for each time step, the simulation delivers the temporal evolution of all the different processes and the temporal change of the limiter surface composition.

As an example Figs 3-5 show several surface informations for a simulation in which an originally pure tungsten limiter is exposed to the SOL plasma under typical ohmic conditions. The input 

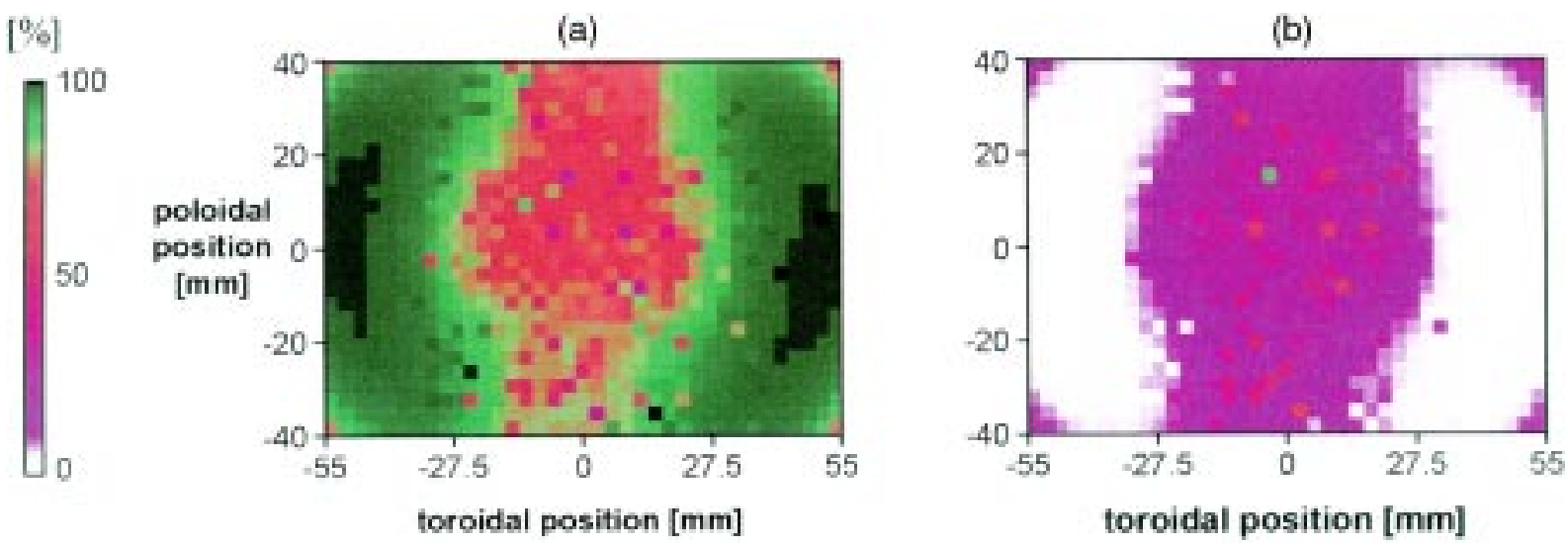

Figure 3. Distribution of (a) carbon and (b) tungsten inside the interaction layer of an originally pure tungsten limiter after having reached equilibrium. The pictures show a plan view of the limiter. The simulation parameters used (Table 1) represent a typical ohmic shot of TEXTOR-94.

parameters used for this simulation are summarized in Table 1. The figures represent the situation after which equilibrium is reached so that the surface composition in the interaction layer does not change anymore with increasing exposure time. Areas in Fig. 3 where the carbon concentration has reached $100 \%$ (at the edges of the limiter) are net deposition zones: here a carbon layer is built up having a thickness which, of course, increases continuously with increasing exposure time. At the locations where the carbon concentration is less than $100 \%$, the incoming carbon flux (sum of background flux and redeposition) is in balance with the outgoing carbon flux, only the tungsten in these regions suffers a continuous erosion. The buildup of net deposition zones at the toroidal edges and a net erosion zone in the middle part of the limiter surface is in agreement with experimental observations [25]. The local erosion and redeposition properties of the limiter after equilibrium has been reached are shown in Fig. 4 for tungsten and in Fig. 5 for carbon. Due to the longer ionization length of carbon the redeposition rate of eroded carbon particles is clearly smaller than that of eroded tungsten particles. In addition the smaller gyration radius of carbon reduces the probability of prompt redeposition. The amount of prompt redeposition is determined by the ratio $P$ of the ionization length to the gyration radius. The plasma conditions considered result in a $P$ value of about 4 for tungsten whereas the $P$ value of carbon is much higher (about 100). To see this difference quantitatively the redeposition rates for carbon and tungsten averaged over the whole limiter surface are summarized in Table 2.
Table 1. Summary of the input parameters used for the simulation of a typical ohmic shot at TEXTOR-94

\begin{tabular}{|c|c|}
\hline Limiter & $\begin{array}{ll}\text { Material: } & \text { tungsten } \\
\text { Shape: } & \text { sphere with radius }=70 \mathrm{~mm} \\
& \text { poloidal length }=80 \mathrm{~mm} \\
& \text { toroidal length }=110 \mathrm{~mm} \\
& \text { interaction depth }=20 \mathrm{~nm}\end{array}$ \\
\hline Magnetic field & $\begin{array}{l}2.25 \mathrm{~T} \\
\text { LCFS at limiter tip }\end{array}$ \\
\hline Plasma & $\begin{array}{l}T_{e}(\mathrm{LCFS})=T_{i}(\mathrm{LCFS})=40 \mathrm{eV} \\
n_{e}(\mathrm{LCFS})=4 \times 10^{12} \mathrm{~cm}^{-3} \\
\text { Fuel ions: deuterium, } Q=1 \\
\text { Impurities: } 2 \% \text { carbon, } Q=4 \\
\\
1 \% \text { oxygen, } Q=5\end{array}$ \\
\hline Decay lengths & $\begin{array}{l}\lambda_{T_{e}}=\lambda_{T_{i}}=25 \mathrm{~mm} \\
\lambda_{n_{e}}=20 \mathrm{~mm} \\
\lambda_{C}=\lambda_{O}=-70 \mathrm{~mm}\end{array}$ \\
\hline $\begin{array}{l}\text { Plasma-surface } \\
\text { interaction }\end{array}$ & $\begin{array}{l}\text { Physical sputtering } \\
\text { Carbon deposition from background }\end{array}$ \\
\hline $\begin{array}{l}\text { Time resolution } \\
\text { of the simulation }\end{array}$ & Time step, $\Delta t=0.2 \mathrm{~s}$ \\
\hline
\end{tabular}

The ERO-TEXTOR code provides further information. First, the energy and charge distributions of redeposited particles are calculated. For the above described simulation the results are presented in Fig. 6. On average the prompt redeposited particles have smaller charges as well as lower energies because of a shorter confinement inside the plasma. 


\section{A. Kirschner et al.}
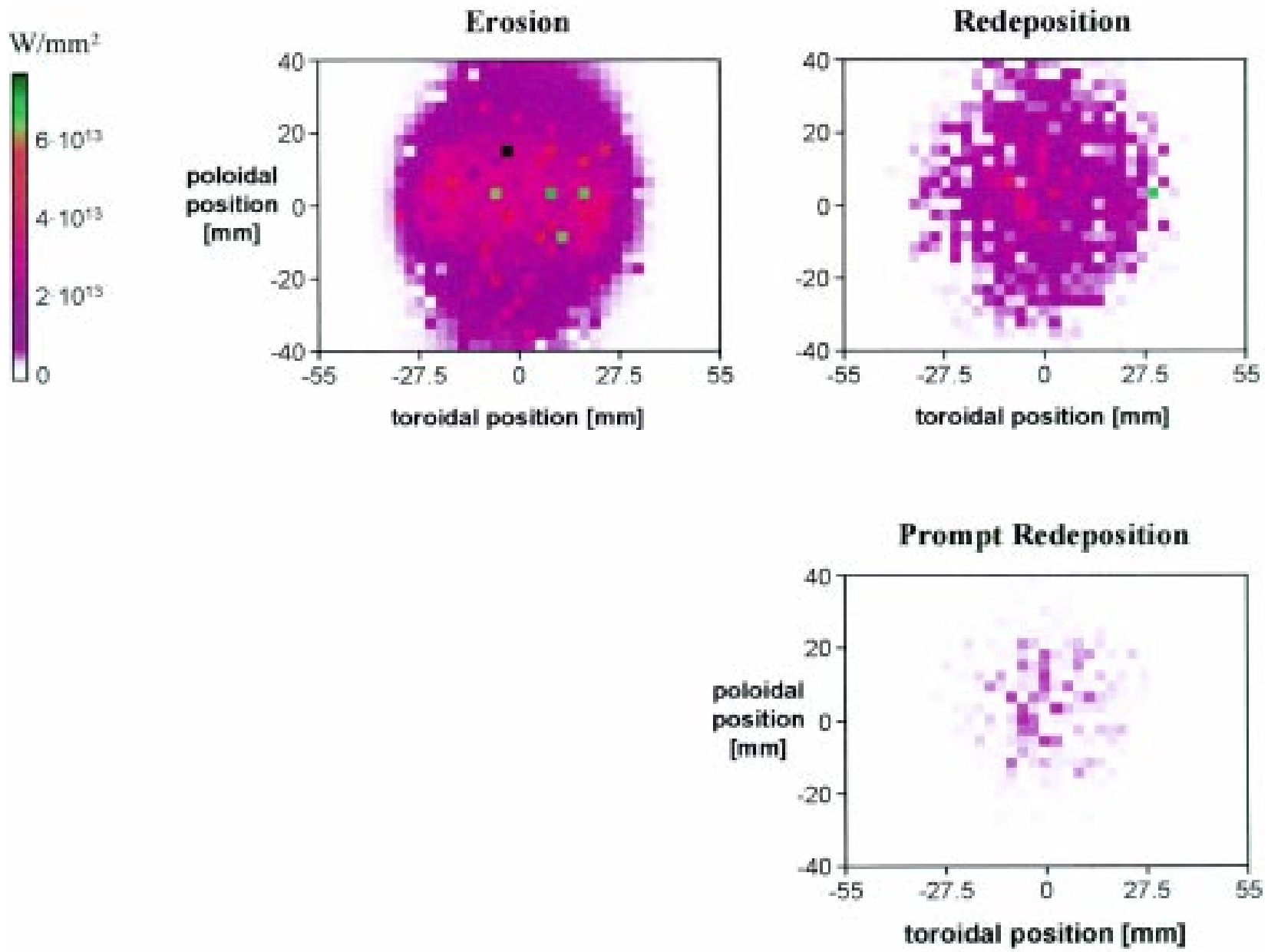

Figure 4. Tungsten erosion and redeposition of the originally pure tungsten limiter after having reached equilibrium (the simulation parameters are given in Table 1). The pictures show a plan view of the limiter integrated over a time of $0.2 \mathrm{~s}$.

In addition, using given material parameters for the heat conductivity and heat capacity, the code calculates the temperature of the limiter achieved after each time step and for each surface cell.

\subsection{Spectroscopic information}

The ERO-TEXTOR code can determine the density of a certain species with charge $Q$ integrated along a certain viewing line for each time step. The spatial resolution for these data is given by the user, who defines the net volume for spectroscopy. The integration of the densities can be carried out along the poloidal, toroidal or radial direction. The direction of integration represents the viewing direction of the spectrometer used under given experimental conditions in TEXTOR-94. From the calculated density of a certain atomic or molecular state the intensity of a certain spectroscopic line emission is obtained by multiplying this density at each location with the electron density at this location and the local rate coefficient of emission of the given species.

As an example Fig. 7(a) shows the densities of neutral tungsten $\mathrm{W}^{0}$ and carbon $\mathrm{C}^{0}$ for the simulation described above under conditions where equilibrium is reached. The integration is carried out along the poloidal direction. In Fig. 7(b) the corresponding toroidal and radial profiles of the $\mathrm{W}^{0}$ and $\mathrm{C}^{0}$ densities are given. Such pictures show directly the penetration length of different species into the plasma. 

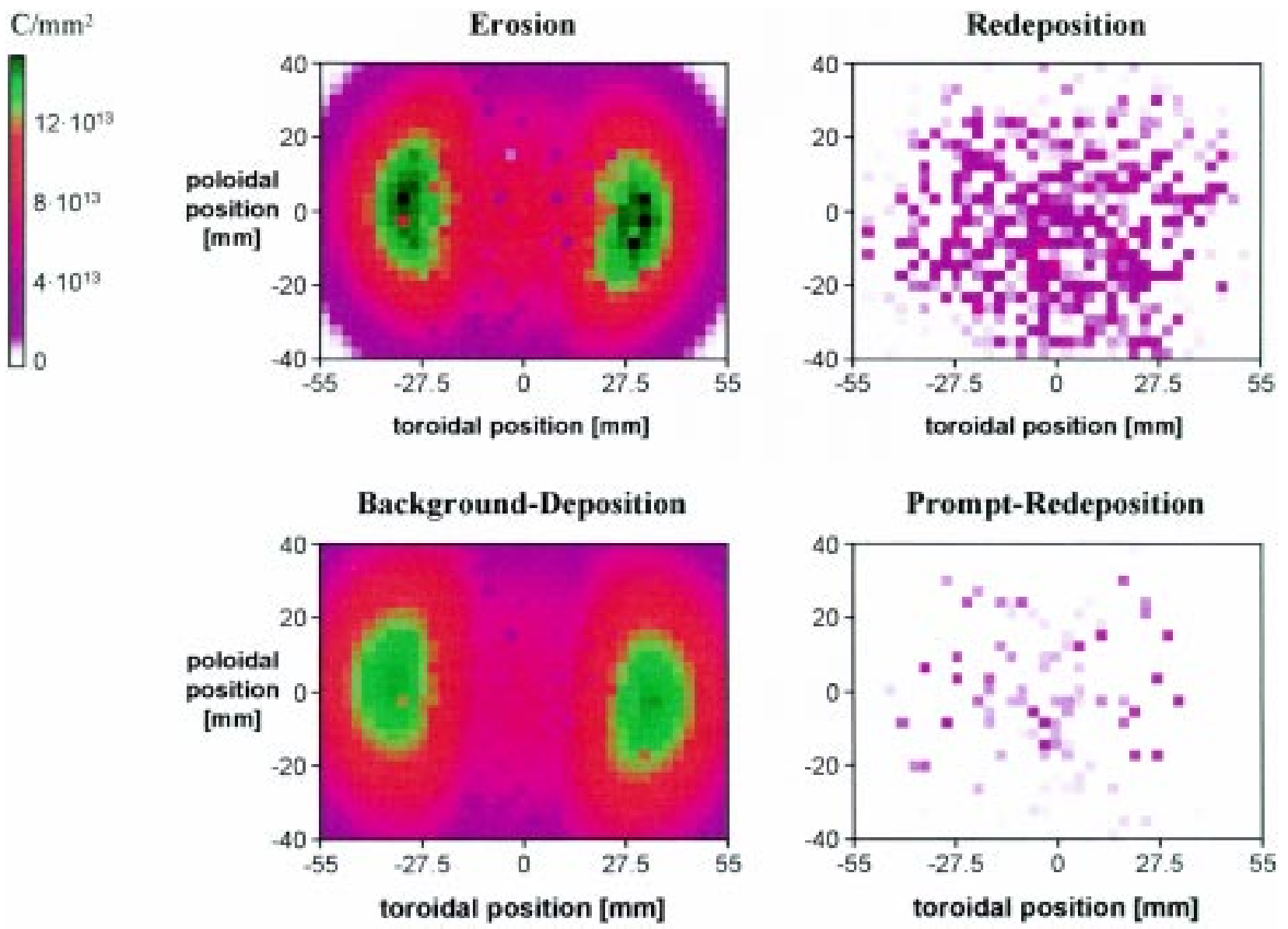

Figure 5. Carbon erosion and redeposition of the originally pure tungsten limiter after having reached equilibrium (the simulation parameters are given in Table 1). The pictures show a plan view of the limiter integrated over a time of $0.2 \mathrm{~s}$.

Table 2. Redeposition rates for tungsten and carbon averaged over the limiter surface

\begin{tabular}{lcc}
\hline & $\begin{array}{c}\text { Tungsten } \\
(\%)\end{array}$ & $\begin{array}{c}\text { Carbon } \\
(\%)\end{array}$ \\
\hline Total redeposition & 36 & 8 \\
Prompt redeposition related & 74 & 14 \\
$\quad$ to the total redeposition & & \\
\hline
\end{tabular}

\section{Brief comparison between ERO-TEXTOR simulations and experimental results}

The main aim of the present article is to demonstrate the ERO-TEXTOR code. Nevertheless, to proof the capability of simulating real physics, in the following some results for tungsten test limiters (spherically shaped surface, Table 1) obtained with the ERO-TEXTOR code are compared with experimental findings at TEXTOR-94. The discussion is limited to the observation of the WI line emission from neutral tungsten atoms $\mathrm{W}^{0}$ and the CII line emission resulting from charged carbon $\mathrm{C}^{+}$ions near the limiter. Experimentally the radial dependence of the emission is observed by means of a spectrometer positioned at a fixed toroidal position (about $2 \mathrm{~cm}$ away from the limiter tip). The intensities are integrated in the poloidal direction. As one example Fig. 8 shows the simulated and measured radial profiles of WI emission for $T_{e}(\mathrm{LCFS})=80 \mathrm{eV}$ and $n_{e}(\mathrm{LCFS})=4.8 \times 10^{12} \mathrm{~cm}^{-3}$, where the LCFS is positioned at the radial position of the limiter tip. The simulation is in good agreement with the experiment. To investigate the influence of the plasma conditions the $e$-folding decay length $\lambda_{W I}$ of the profiles is determined for increasing electron densities. In Fig. 9 the 
(a)
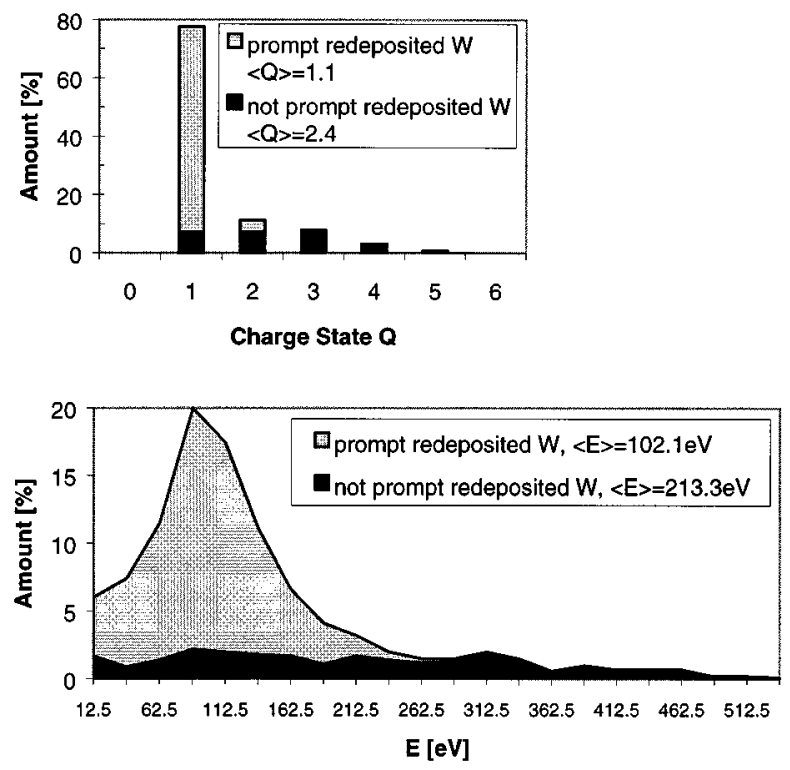

(b)
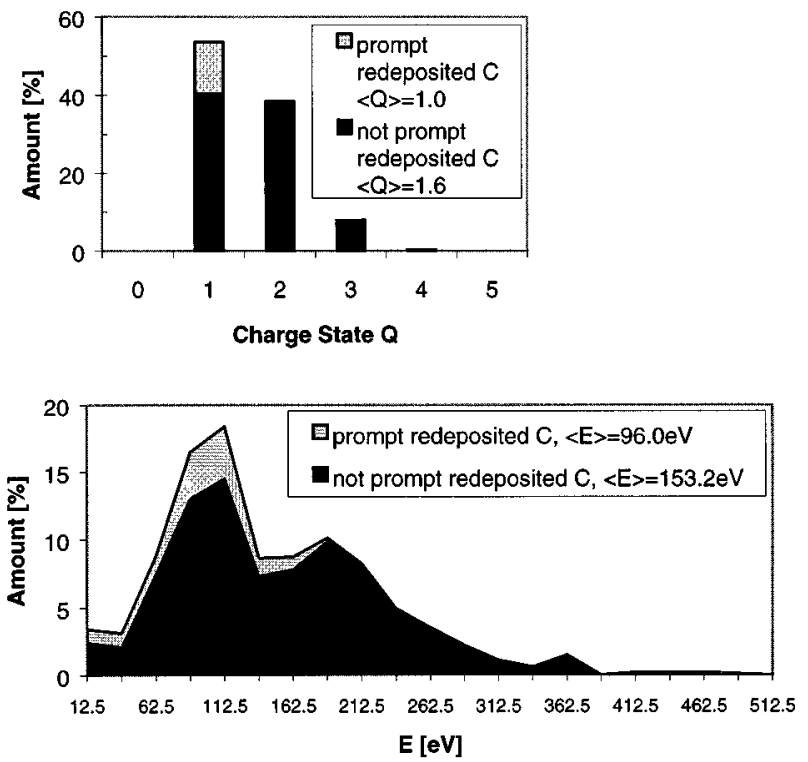

Figure 6. (a) Distribution of the charge state $Q$ and energy $E$ of redeposited tungsten particles at a time when the surface composition has reached equilibrium. (b) As (a) but for carbon.

experimentally obtained $\lambda_{W I}$ is compared with simulated values for densities between $4.0 \times 10^{12} \mathrm{~cm}^{-3}$ and $1.5 \times 10^{13} \mathrm{~cm}^{-3}$. The electron temperature varies for these densities between 90 and $30 \mathrm{eV}$. The comparison between experiment and simulation results in a good agreement. To compare the experimental and simulated radial profiles of the CII emission, the above plasma conditions $\left(80 \mathrm{eV}, 4.8 \times 10^{12} \mathrm{~cm}^{-3}\right)$ are used. The simulated CII profile consists of two parts. First, there is a contribution from sputtered carbon. Second, the carbon ions from the background plasma which are reflected at the limiter surface (as neutrals) contribute to the CII profile above the limiter. Figure 10 presents the measured and simulated profiles. Again a comparison results in a good agreement. In particular, the simulation is able to reproduce a nonzero CII intensity relatively deep into the plasma due to reflected background carbon ions. Finally, Fig. 11 presents the influence of the plasma conditions on the $e$-folding length $\lambda_{C I I}$ of the CII profile, where the simulated values correspond well with the measured ones.

The good agreement of the simulated radial line emission profiles with the measured ones indicates that the ERO-TEXTOR code describes correctly the transport of eroded particles through the edge plasma.

\section{Summary}

The three dimensional Monte Carlo code EROTEXTOR was developed to simulate the erosion and redeposition behaviour of test limiters exposed to the scrape-off layer of TEXTOR-94. The background plasma is described in the code by a one dimensional plasma model. The radial profiles of the temperatures (electrons and ions) and densities (electrons and impurities) in the edge plasma are taken from experimental data and are thus input parameters. The transport of impurities through the plasma is given by the forces due to the magnetic and electric fields as well as friction, diffusion and thermal force effects. The ionization and dissociation probabilities are determined using the Monte Carlo method. Different plasma-wall interaction processes (physical and chemical erosion, reflection) are treated using published data. As a result the code delivers much output information for each calculation step. In particular, spectroscopic data and the surface composition of a limiter after exposure can be compared with measurements.

In fusion research the lifetime of plasma facing components is one of the most important problems to be solved. The ERO-TEXTOR code is able to deliver predictions concerning the lifetime of different 


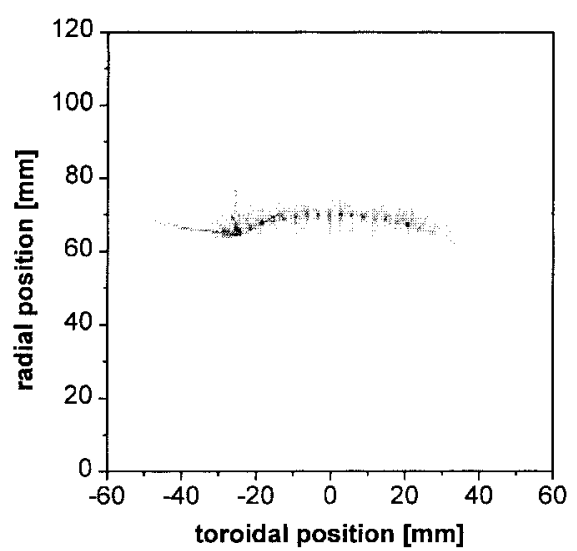

(a)

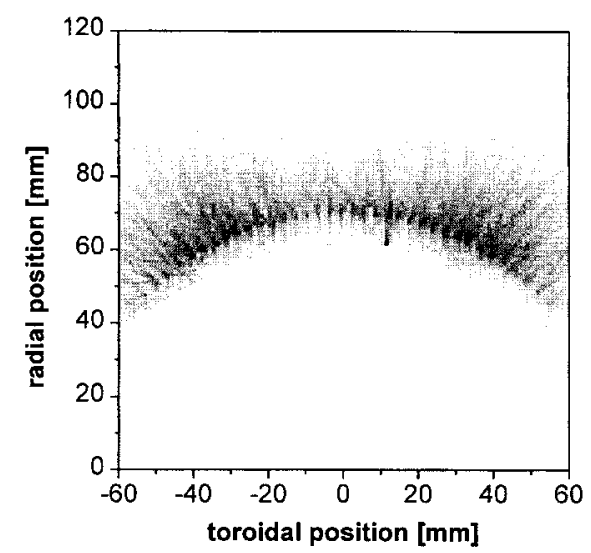

(b)
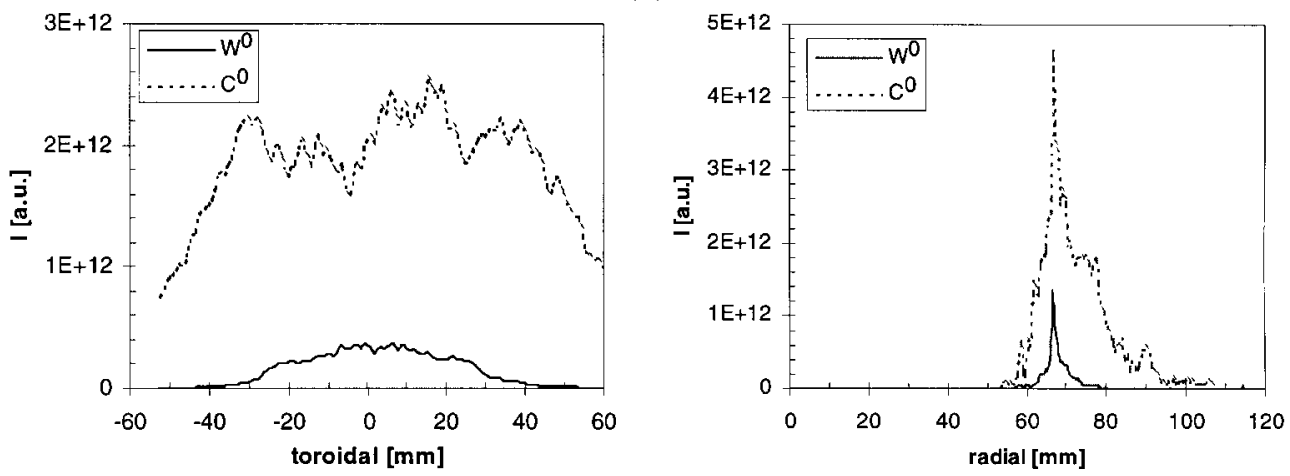

Figure 7. (a) Density of neutral tungsten (left hand side) and neutral carbon (right hand side) above the limiter integrated over the poloidal direction. Again the pictures represent a time point where equilibrium is reached for the simulation of the tungsten limiter described above (colour scaling for both pictures $0-255$ ). (b) Profiles of the $\mathrm{W}^{0}$ and $\mathrm{C}^{0}$ densities in the toroidal direction taken at $r=r_{\text {LCFS }}=r_{\text {Limitertip }}=70 \mathrm{~mm}$ (left hand side) and in the radial direction taken at toroidal $=20 \mathrm{~mm}$ (right hand side).

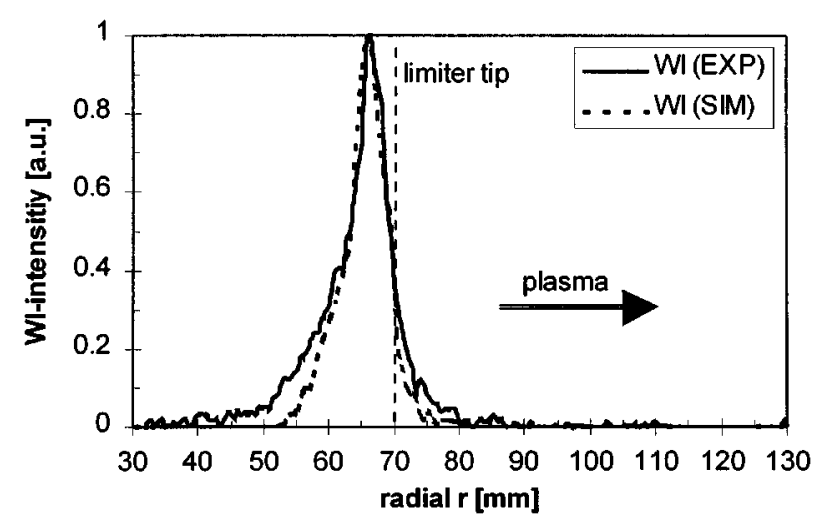

Figure 8. Simulated and measured normed radial profiles of the WI emission above a tungsten test limiter exposed to a plasma with $n_{e}(\mathrm{LCFS})=4.8 \times 10^{12} \mathrm{~cm}^{-3}$ and $T_{e}(\mathrm{LCFS})=80 \mathrm{eV}$.

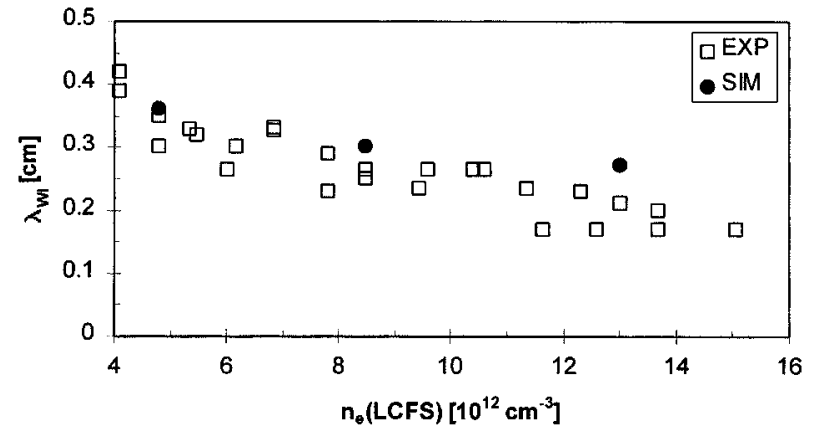

Figure 9. Simulated and measured $e$-folding decay lengths $\lambda_{W I}$ of the radial WI profile as a function of the electron density. The electron temperature varies between 90 and $30 \mathrm{eV}$. 


\section{A. Kirschner et al.}

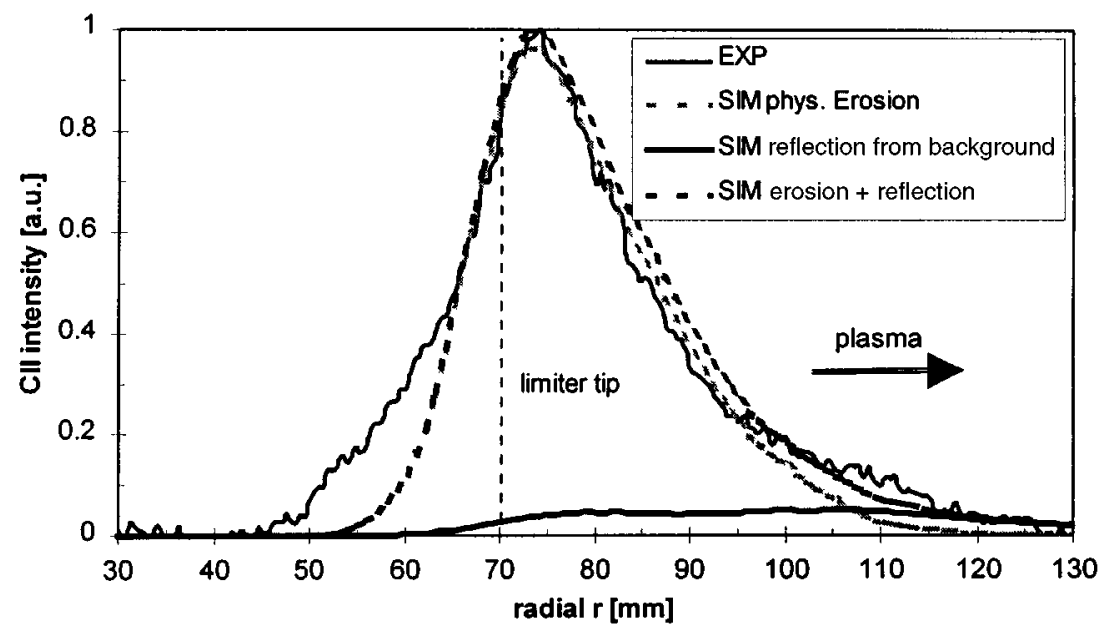

Figure 10. Simulated and measured normed radial profiles of the CII emission above a tungsten test limiter exposed to a plasma with $n_{e}(\mathrm{LCFS})=4.8 \times 10^{12} \mathrm{~cm}^{-3}$ and $T_{e}(\mathrm{LCFS})=80 \mathrm{eV}$.

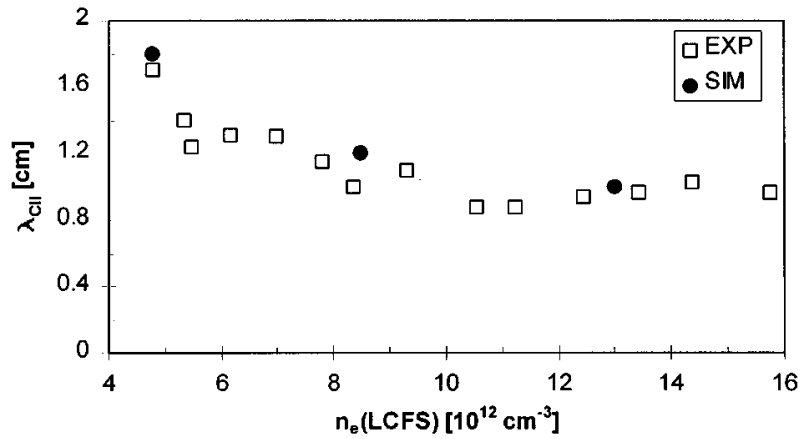

Figure 11. Simulated and measured $e$-folding decay lengths $\lambda_{C I I}$ of the radial CII profile as a function of the electron density. The electron temperature varies between 90 and $30 \mathrm{eV}$.

materials under various plasma conditions. In addition, combining the ERO-TEXTOR results of the net erosion of wall elements with a 'core transport code', it is possible to make statements about the dilution of the core plasma due to eroded material. Moreover first attempts at implementing the divertor geometry in the ERO-TEXTOR code have been completed. Therefore in the near future it will be possible to produce plasma-wall interaction simulations which are directly relevant to ITER.

\section{References}

[1] Naujoks, D., Behrisch, R., Coad, J.P., de Kock, L., Nucl. Fusion 33 (1993) 581.
[2] Stangeby, P.C., McCracken, G.M., Nucl. Fusion 30 (1990) 1225.

[3] Stangeby, P.C., Elder, J.D., Nucl. Fusion 35 (1995) 1391.

[4] Stangeby, P.C., The Plasma Sheath in Physics of Plasma Wall Interaction in Controlled Fusion (Post, D.E., Behrisch, R., Eds), NATO ASI Series, Plenum Press, New York and London (1986).

[5] Chodura, R., Phys. Fluids 25 (1982) 1628.

[6] Brooks, J., Phys. Fluids B 2 (1990) 1858.

[7] Tokar', M.Z., Contrib. Plasma Phys. 36 (1990) 250.

[8] Eckstein, W., Garcia-Rosales, C., Roth, J., Ottenberger, W., Sputtering Data, Rep. IPP9/82, MaxPlanck-Institut für Plasmaphysik, Garching (1993).

[9] Yamamura, Y., Itikawa, Y., Itoh, N., Rep. IPPJAM-26, NIFS, Nagoya (1983).

[10] Abramov, V.A., Igitkhanov, Y.L., Pistunovich, V.I., Pozharov, V.A., J. Nucl. Mater. 162-164 (1989) 462.

[11] Eckstein, W., Philipps, V., Physical Sputtering and Radiation Enhanced Sublimation, Rep. KFA-IPP 02/96, KFA-IPP, Jülich (1996).

[12] Roth, J., García-Rosales, C., Nucl. Fusion 37 (1997) 897.

[13] Refke, A., Philipps, V., Vietzke, E., J. Nucl. Mater. 250 (1997) 13.

[14] Eckstein, W., Computer Simulation of Ion-Solid Interaction, Springer-Verlag, Berlin (1991).

[15] Knauf, H., Numerische Modellierung von Rückstreuung und Desorption an Wandoberflächen zur Interpretation von spektroskopischen Messungen in der Plasmarandschicht von Tokamaks, Rep. Jül3415, Forschungszentrum Jülich (1997). 
[16] Bell, K.L., et al., Atomic and Molecular Data for Fusion, Part I, Rep. CLM-R216 Culham Laboratory, Abingdon (1982).

[17] Ehrhardt, A.B., Langer, W.D., Collisional Processes of Hydrocarbons in Hydrogen Plasmas, Rep. PPPL2477, Princeton Plasma Phys. Lab., NJ (1986).

[18] Chatam, H., Hills, D., Robertson, R., Gallagher, A., J. Chem. Phys. 81 (1984) 1770.

[19] Perrin, J., et al., Chem. Phys. 73 (1982) 383.

[20] Birdsall, C.K., Plasma Physics via Computer Simulation, McGraw-Hill, New York (1985).

[21] Montgomery, D.C., Tidman, D.A., Plasma Kinetic Theory, McGraw-Hill, New York (1964).

[22] Spitzer, L., Physics of Fully Ionized Gases, Wiley, New York (1962).
[23] Braginskii, S.I., Transport Processes in Plasmas, Reviews of Plasma Physics, Vol. 1, Consultants Bureau, New York (1965).

[24] Kögler, U., et al., J. Nucl. Mater. 241-243 (1997) 816.

[25] Philipps, V., et al., J. Nucl. Mater. 258-263 (1998) 858.

(Manuscript received 25 May 1999

Final manuscript accepted 24 February 2000)

E-mail address of A. Kirschner:

a.kirschner@fz-juelich.de

Subject classification: I2, Tm 